\title{
Challenges to optimizing a telescope system to detect and characterize exo-solar planetary systems
}

James B. Breckinridge

James B. Breckinridge, "Challenges to optimizing a telescope system to detect and characterize exo-solar planetary systems," Proc. SPIE 5875, Novel Optical Systems Design and Optimization VIII, 587508 (29 August 2005); doi: 10.1117/12.619697 


\title{
Challenges to optimizing a telescope system To detect and characterize exo-solar planetary systems
}

\author{
James B. Breckinridge \\ Jet Propulsion Laboratory ${ }^{1}$ \\ California Institute of Technology \\ Pasadena, CA. 91109
}

\begin{abstract}
Novel optical design and engineering ideas are needed to build the large space telescopes for the direct detection and characterization of exo-solar system planets. For example, the Terrestrial Planet Finder Coronagraph requires a primary mirror $4 \times 8$ meters in size that is $>10 \mathrm{x}$ smoother than the 2.8 meter HST mirror and have a uniform reflectivity across the mirror to within $0.1 \%$. The telescope system will need to control scattered light to within a part in 10 billion. The Terrestrial Planet Finder Interferometer will be a white-light, broadband infrared interferometer with a baseline in excess of 50 meters.

In addition to direct imaging, planets masses and orbits can be derived from very precise measurements of the position of a star as it moves across the background. Interferometers provide the highest accuracy measurements of relative positions

We will show that the optical design and the mechanical layout \& configuration for these new telescopes need to be optimized for polarization as well as scattered light. Material science and coating technology plays an important role in the optimization of these systems. Stress across the surface of a mirror and stress within the optical thin film introduces polarization dependent scattered light. A new method to measure the anisotropy of the polarization-reflectivity of thin metal films on large astronomical mirrors is described.
\end{abstract}

\section{INTRODUCTION}

Two of the most compelling scientific questions today: Are we alone? And how did we get here?, require the discovery and characterization of exo-solar planetary and protoplanetary systems. NASA is currently developing three space observatories and planning for three more for launch after 2025 to answer these questions. The three observatories under development are the Space Interferometer Mission (SIM), the Kepler Mission (KM), the Terrestrial Planet Finder-Coronagraph (TPF-C) mission and the Terrestrial Planet Finder-Interferometer (TPF-I) mission. Each of these three will search for planets, with its own unique telescope \& instrument approach. The SIM is a metrology observatory that measures, with incredible precision, the motion through our galaxy of about 2,000 candidate stars, searching each one for the characteristic periodic "wobble" that results from the reflexive motion of a planet too faint to be seen in orbit about the much brighter star. The precision of this instrument is similar to that needed to measure the thickness of a dime inserted edge-on into the sand on the surface of the moon!

The Kepler is a precision radiometer observatory, looking at several thousand stars within a small field of view, to search for a change in the brightness of a few stars as small as one part in ten thousand. The brightness change is expected when a planet in orbit about any star passes in front of its much brighter parent star. This is similar to the recent eclipse of the planet Venus across the disk of our sun. The Terrestrial Planet Finder-Coronagraph (TPF-C) will be the first observatory to make a direct detection of an exo-planetary system by blocking out the very intense light of the parent star to record directly the light reflecting from an exo-solar system planet, which is a hundred billion (1.E-11) times fainter than its parent star. For this very unique telescope we must control the surface errors in the wavefront to unprecedented

\footnotetext{
${ }^{1}$ Work performed at the California Institute of Technology's Jet Propulsion Laboratory under contract with the National Aeronautics and Space Administration (NASA). Proceedings of SPIE Vol. 5875 (SPIE, Bellingham, WA, 2005) · 0277-786X/05/\$15 - doi: 10.1117/12.619697
} 
levels. If the wavefront from TPF-C is expanded to cover the Pacific Ocean, the allowable distortion is 5 microns. This level of smoothness is probably beyond fabrication methodologies, and special purpose adaptive optics is used to control and remove phase and amplitude imperfections. New ingenious approaches are required.

The three planet detection and characterization missions of the future are The Terrestrial Planet Finder Interferometer (TPF-I), The Life Finder mission and The Planet Imager mission. The TPF-I will be a 50 to 100 meter base line infrared (2 to 16 micrometers wavelength) interferometer to explore for Oxygen, Nitrogen, Methane and Ozone in the atmospheres of the exo-planets. The very long baseline will enable searches for planets at a distance from our solar system, 10 times further out than TPF-C's capability. If planets are uniformly distributed, in the neighborhood of the sun, this longer baseline will increase the number of candidate stars by a factor of 100. The Life Finder will carry sensitive spectrometer systems to further examine the chemical composition of the atmosphere of exo-planetary systems to detect and characterizes gases in the atmosphere, in particular oxygen, nitrogen, methane, those gases we know support life as we know it here on earth.

What is it that characterizes the optical instruments that we need to build to enter this new field of science to search for life beyond our solar system? Telescopes larger than ever before - to collect the photons \& have high angular resolution. And telescopes more stable than ever before. Pointing, tracking, image quality, scattered light control, polarization properties of the optics, and new highly reflective coatings present technical barriers that we will overcome.

In this paper we will review the need that these new planet-finding engines have for innovative optical design, engineering and optical coatings.

\section{WHAT IS IT WE ARE TRYING TO IMAGE?}

Theoretical models of the stellar and planetary formation process indicate that high angular resolution astronomical imaging systems will let us image the process of the formation of stars and planets from the gas and dust so common within the Universe. Figure 1, shown below, shows the results of four theoretical and numerical modeling efforts to understand the formation of proto-planetary systems and solar nebula models. These figures are taken from 4 science peer-reviewed papers.
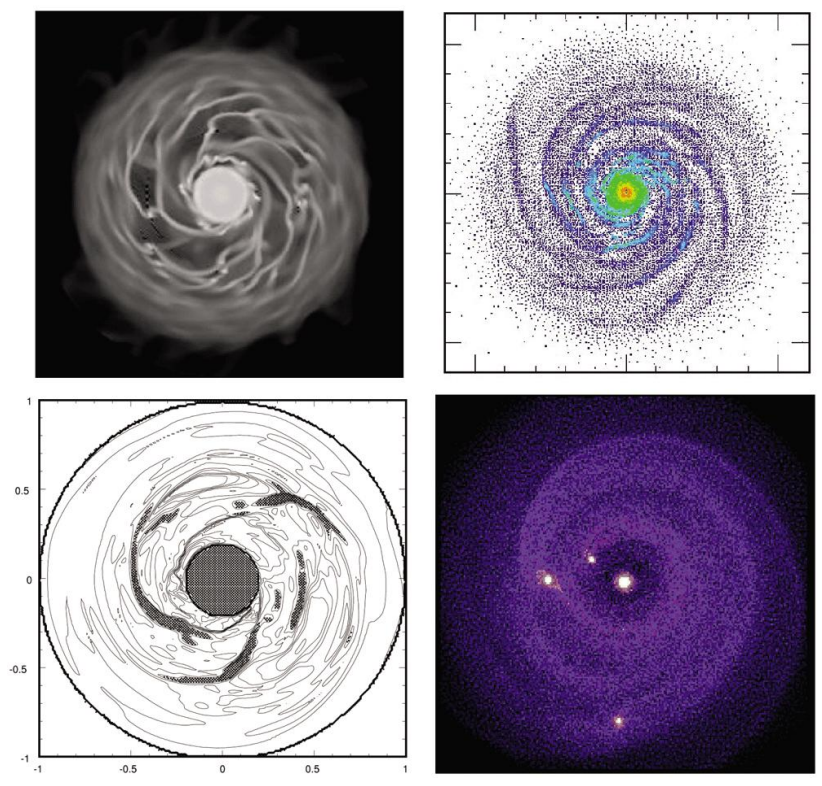
Figure 1 shows the results of four theoretical and numerical modeling efforts to understand the formation of proto-planetary systems and solar nebula models. Several simulations of planetary formation Pickett ET. Al. (from reference 1) are shown

Based on these theoretical models, and similar ones completed earlier prompted Hubble Space Telescope (HST) astronomers to added field stops and special tapered coatings to their HST instruments which enabled the first high resolution direct images of stellar and possibly protoplanetary system formation.

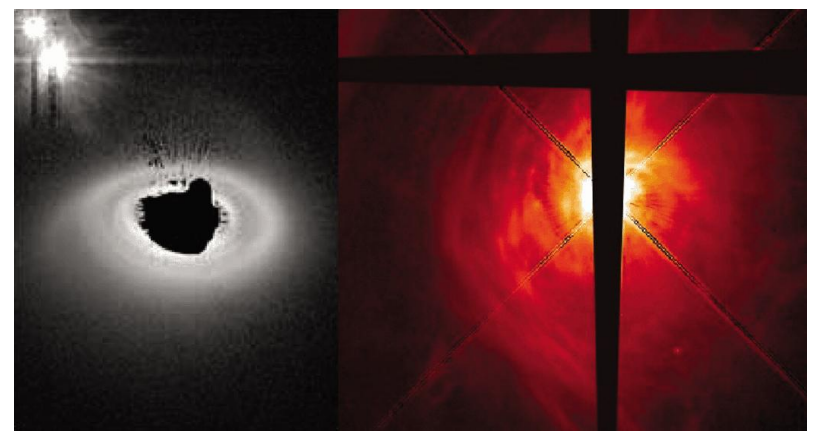

Figure 2. HST images showing faint dust and gas clouds around stars believed to indicate a planet formation process. (NASA, Brady/Woodgate/Bruhweiler/Bogess/Platt/Lindler, images from Pickett and $\operatorname{Lim}^{1}$

\section{ASTRONOMICAL CORONAGRAPHS}

Solar astronomers regularly use coronagraphs to study the very faint corona of the sun and solar prominences, as they appear just above and close to the extremely bright solar disk. Ground-based coronagraph-telescopes control scattered light to within a part in ten to the $6^{\text {th }}$. Scattering in the earth's atmosphere, even at the great elevations of these solar-coronagraph observatories provides a natural limit of only about one part in ten to the 7, and only then on rare occasions. Figure 3, below, shows a schematic for a coronagraph.

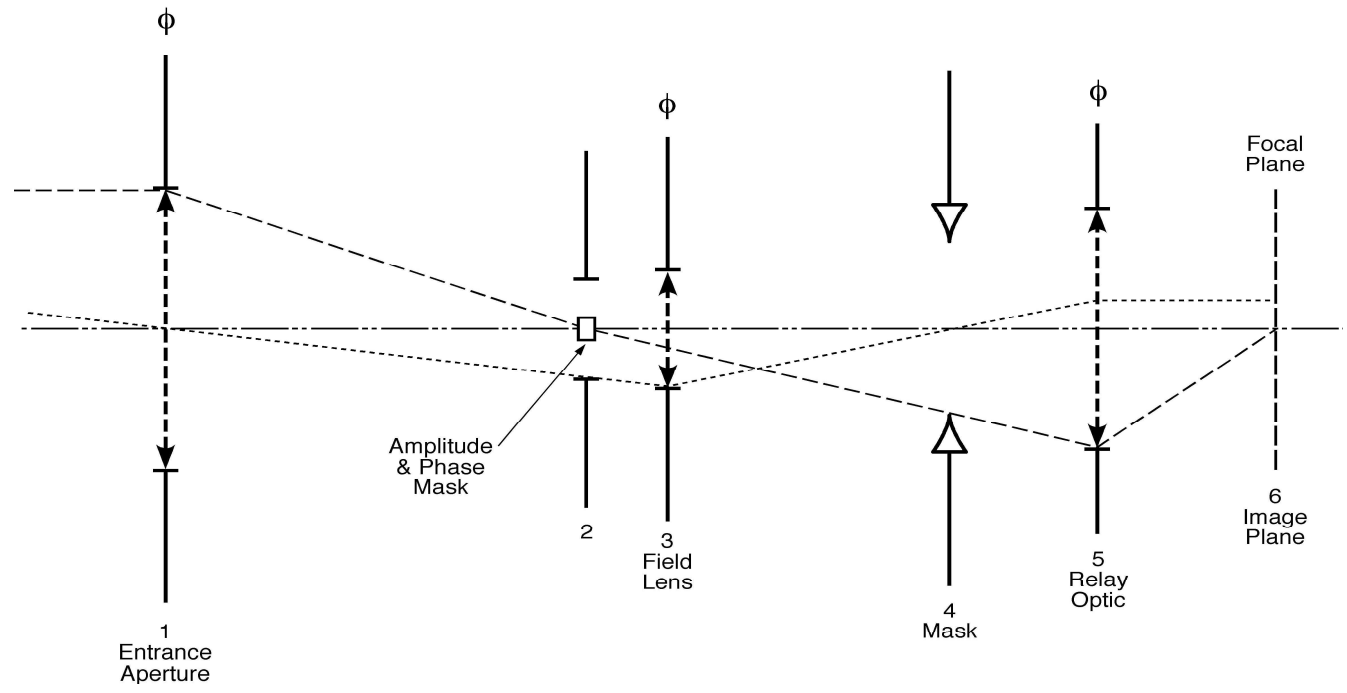

Figure 3 Schematic of a high-performance coronagraph. 
A high performance coronagraph is shown in Figure 3. Light from a distant object (sun or a star) enters the entrance aperture at plane 1 of optical power $\Phi_{1}$ from the left in the drawing above. An image of object space is formed at an image plane located at plane 2 where an amplitude and phase mask is positioned to block out the bright star in the field. The telescope is centered with the star on axis. The dimensions of this spot, that is its size, amplitude, phase transmittance and reflectance profiles are optimized to control the amount of unwanted radiation passed to the system. If the focal length is short, then the spot may be well less than 500 microns across. To the right of this image plane mask at plane 3 is located a field lens of optical power $\Phi_{3}$. This field lens relays an image of the entrance aperture at plane 1 onto an edge-mask at plane 4. In some cases plane 2 is positioned at the focus of the powered element 3. This will collimate the light from plane 2. Returning to plane 1: diffraction around the edge of the entrance aperture at plane 1 creates a bright ring of unwanted radiation at the image of the entrance aperture. The ring mask at plane 4 is designed and built to control the amplitude and phase transmittance of the unwanted radiation diffracted around the edge of the entrance aperture at plane 1 to maximize the probability of detection. The optic at plane 5 relays the image plane 2 onto the final image plane 6 where the detector array is located.

Most early solar coronagraphs used a simple metal stop shaped to match the curvature of the sun's limb at the image plane, to control scattered light (unwanted radiation) from passing further into the telescope than the first image plane. The most effective solar coronagraphs took advantage of the fact that solar prominences appear distinct in a Hydrogen emission line designated as $\mathrm{H}$-alpha and emitting at $656 \mathrm{~nm}$ wavelength. Therefore they could use a single refractive, bi-convex optical element, of high quality glass (e.g. BK7) whose curvatures were optimized to minimize spherical aberration, and provide highacuity at the image plane. Because of uncorrectable longitudinal chromatic aberration, solar coronagraphs used to observe the $\mathrm{Ca}$ Fraunhofer $\mathrm{H} \& \mathrm{~K}$ lines in the deep blue requires an objective lens different from that used for the deep red line at $\mathrm{H}$-alpha.

Ray Smartt ${ }^{2}$, at the Sacramento Peak Observatory analyzed a reflecting solar coronagraph. These reflecting solar coronagraphs have scattered light greater than that found in refractive coronagraphs, but are sufficient to observe the broadband radiation emitted by the outer solar corona. In some research applications the broad spectral-band, white light coverage provided by the mirrored reflecting surface out weighs the increase in unwanted radiation.

\section{CHALLENGES TO BUILDING EXO-PLANET IMAGING STELLAR CORONAGRAPHS}

The stellar coronagraph is far more technologically challenging than the solar coronagraph in several aspects.

Stars that are candidates to have planets around them are between ten to the $10^{\text {th }}$ and 10 to the $15^{\text {th }}$ times fainter than our sun. And exo-planets, shining in the reflected sunlight of the planetary system's parent star, are much fainter than that by another factor of ten to the $10^{\text {th }}$ to ten to the $15^{\text {th }}$. Telescope apertures in the range of 8-meters are required to collect sufficient number of signal photons in the presence of the very bright central star. Innovative optical designs and optical materials research plays an important role in the development of very high contrast (Hyper-Contrast Optical Systems).

Current work under way to minimize unwanted radiation in a planet-finding coronagraph includes, A. small excursion adaptive optics to clean-up white-light speckles at the image plane ${ }^{3}{ }^{4}$, B. polarization analyses and measurements, and C. engineering design and technology development ${ }^{5}$.

\section{POLARIZATION EFFECTS IN TELESCOPES}

Breckinridge and Oppenheimer ${ }^{6}$ and Breckinridge ${ }^{7}$ identified two sources of polarization induced scattered light in hyper-contrast optical systems. These two sources are the tilted metal mirrors within large telescope systems and the non-uniformity of highly reflecting thin metal films that coat the mirrors of the 
high performance telescope. They calculated the polarization transmittance of a reflecting coronagraph and determined that the image quality is instrumentally introduced polarization dependent and suggested ways to mitigate these deleterious effects.

To further complicate matters the gas and dust that coalesce to form planets provide important polarization information to understand the physics of planet formation. The radiation entering the coronagraph is slightly polarized with a signature that shoukd be retrieved to understand planet formation.

It is well known that in order to have an interferometer form fringes, the two beams must be coherent with each other. Image formation is an interferometric process. If the two beams are not of the same polarization no fringes are observable. The same is true for the image formation process in a telescope. If one performs the experiment of covering one half of the telescope entrance aperture with a polarizer transmitting in one polarization state and the other half covered with a polarizer transmitting in a polarization state orthogonal to the first the point spread function of the system is a algebraic sum of two "D" shaped point spread functions. Radiation coming from tall regions of the pupil must be of the same polarization state in order to create diffraction limited optical system with the classic required symmetrical point spread function.

One source is introduced by the fact that incoming beams from the source do not strike the primary mirror at the same angle across the mirror. For example, for an $\mathrm{F} \#=1 \mathrm{system}$, the marginal ray strikes the edge of the concave primary mirror at an angle of almost 20 degrees, whereas the chief ray reflects from the center of the primary mirror at almost normal incidence for telescopes with reasonably small field of view. As the F-number decreases, the marginal ray angle deviation increases, where the marginal ray angle is simply arc tan $(1 / 2 \mathrm{~F})$, where $\mathrm{F}$ is the $\mathrm{F}$-number of the imaging system. In this case there is a spatial dependence of the polarization reflectivity across the pupil. Thos rays striking the edge of the mirror have a greater difference in reflectivity between two orthogonal linear two polarizations than do the rays near the center. The effect of this polarization apodization on the image formation process was examined in detail by R. Chipman ${ }^{8}$. Breckinridge and Oppenheimer ${ }^{5}$ show that the contour or shape of the point-spread function of the telescope depends on instrumentation-induced polarization, in addition to the partial polarization of the source.

The other source is caused by anisotropies in the highly reflecting thin metal film deposited over the precision optically figured glass surface. It has been recognized for years that the scattered light performance of the large primary mirror strongly depends on the smoothness of the mirror substrate and much effort has gone into understanding the engineering processes to create a super-smooth surface. The large area-reflecting surface of these substrates, however, does not have the quality specular reflectance required for the mirror. Thus, the substrate must be coated with the highly reflecting metal thin film. If this thin metal film is perfectly isotropic then the limiting effects discussed in section 2 will dominate. Unfortunately, metal thin films over the large areas ( $\sim 60$ meters square) required for astronomy are not, in general isotropic.

A qualitative understanding of the effects of metal thin film inhomogenieties on system scatteredlight is provided by analysis of the Smith-Purcell Effect ${ }^{9},{ }^{10}$. An electromagnetic light wave incident on a metal surface is known to induce an oscillating electronic charge inside the highly conducting metal film on the mirror substrate. The period of oscillation is characteristic of the frequency of the source; that is about $10 \mathrm{E} 14 \mathrm{~Hz}$. If this highly conducting metal has perfect isotropic conductance over its entire volume, then the images formed by the mirror will be, "perfect" within the limits of diffraction produced by the edge of the clear aperture. A review and analysis ${ }^{11}$ of the micro structural induced anisotropy in optical thin films demonstrated their presence. Reflection anisotropy ${ }^{12}$ in telescope mirror coatings has been examined. Measurements ${ }^{13}$ of the optical anisotropy in thin films deposited obliquely were made in situ observations and computer models were developed to explain his observations.

Ironically, very little work has gone into optimal deposition of metal thin films onto these supersmooth surfaces (except for small optics used in X-ray imaging devices) ${ }^{14}$. The imperfections in the metal thin films generally manifest themselves in the form of unwanted, usually columnar, structure in the film after it has been deposited on the optical substrate surface.

MEASUREMENT TECHNIQUE FOR A LARGE APERTURE MIRROR. 
Breckinridge $^{7}$ proposed an approach to measuring the polarization reflectivity of large telescope mirrors. Here we discuss that approach and provide additional measurement scope. Figure 4 below shows a diagram of the basic optical test set up to measure the polarization reflectivity across areas of the mirror.

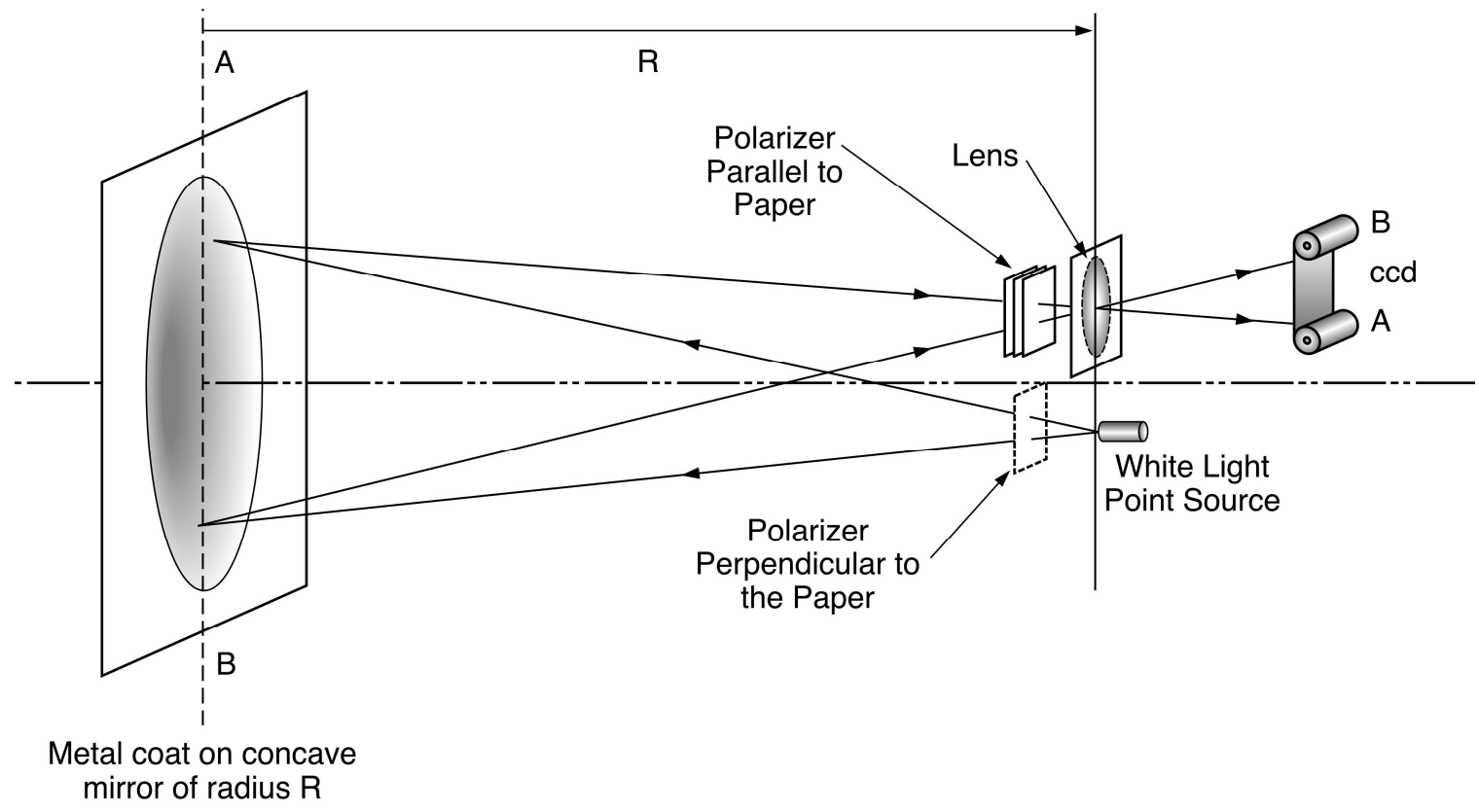

A spherical mirror (AB) coated with a highly reflective metal is located with its center of curvature (at distance $\mathrm{R}$ from the vertex) to the right in the drawing above. Just below the center of curvature we locate the end of a white-light point source for a white light wavefront to fill the aperture of the mirror AB. Alternatively a 100-watt "point-a-light" or vacuum arc light can be used to generate a point source of unpolarized nature. Note that for both the vacuum arc and the fiber bundle, stress birefringence may introduce some circular polarization, but the effect will be small and it may be neglected. The power supply for the white-light source will need to be regulated so the lamp has nearconstant radiant exitence over periods of a few 10's of minutes. The source of radiation should be as close to the center of curvature point as possible so that each ray associated with the spherically expanding wavefront strikes the surface $\mathrm{AB}$ at normal incidence. All rays from the center of curvature strike the large primary mirror at normal incidence. This arrangement ensures that reflectivity anomalies observed across the surface $A B$ will not be the result of the $F \#$ of the system, but rather caused by surface-reflectivity processing effects.

The wavefront expands from the white-light source, fills the mirror surface and reflects back to converge to a point within the lens of a CCD camera located just above the center of curvature. This CCD camera lens re-images the mirror surface $\mathrm{AB}$ (located in the near-field) on to a CCD chip. A polarizer is placed in a stand where it can be rotated about an axis normal to the surface with some precision (0.1degree). Two assemblies of this type are required, one in front of the source that generates the expanding white-light wavefront and the other located in front of and covering the lens of the CCD camera. The focal length of the CCD lens and the front focal distance and back focal distance should be adjusted so a sharp image of the mirror $\mathrm{AB}$ fills the $\mathrm{CCD}$ field of view.

The arrangement described above isolates measurements of thin film polarization anisotropies, because; by going to the center of curvature all rays strike with normal incidence on to the surface. To measure the physical properties of the mirror in the configuration it will be used a collimator (which will introduce its own polarization effects) is required. The use of a collimator will provide information on the uniformity of the real and imaginary parts of the index as revealed by modeling the complex amplitude and phase. In addition, analysis shows that the configuration shown in figure 4, if the white light source is 
replaced with a tunable diode laser, a polarization and phase map of the surface can be obtained, in sufficient detail to use in coronagraph models to predict performance of the image quality.

\section{ACKNOWLEDGEMENTS}

The author acknowledges helpful discussion with John Trauger, Joe Carson and Stuart Shaklan of the Jet Propulsion Laboratory.

This research was carried out at the Jet Propulsion Laboratory, California Institute of Technology, under a contract with the National Aeronautics and Space Administration.

${ }^{1}$ M. K. Pickett and A. J. Lim, Astron. \& Geophysics 45, 1.12-1.17, Feb 2004.

${ }^{2}$ R. N. Smartt, E. W. Cross, Advances in spherical-mirror telescopic systems design: application to large aperture solar coronagraphs, Opt. Engr. 41(8) 2055-2058, 2002.

${ }^{3}$ Trauger, J.T.; Burrows, C.; Gordon, B.; et al. Coronagraph contrast demonstrations with the high-contrast imaging testbed. Optical, Infrared, and Millimeter Space Telescopes, 21-25 June 2005, Glasgow, UK

Proceedings of the SPIE - The International Society for Optical Engineering vol.5487, no.1 : 1330-6, 2004

${ }^{4}$ Lowman, A.E.; Trauger, J.T.; Gordon, B.; et al.

High-contrast imaging testbed for the Terrestrial Planet Finder coronagraph

Optical, Infrared, and Millimeter Space Telescopes, 21-25 June 2005, Glasgow, UK

Proceedings of the SPIE - The International Society for Optical Engineering vol.5487, no.1 : 1246-54, 2004

${ }^{5}$ White, M.L.; Shaklan, S.B.; Lisman, P.D.; et al. Design and performance of the Terrestrial Planet Finder coronagraph, Optical, Infrared, and Millimeter Space Telescopes, 21-25 June 2005, Glasgow, UK

Proceedings of the SPIE - The International Society for Optical Engineering vol.5487, no.1 : 1234-45, 2004

${ }^{6}$ J. B. Breckinridge and B. Oppenheimer (2004), Polarization effects in reflecting coronagraphs for whitelight applications in astronomy, ApJ. $\underline{600}$ 1091-1098.

7 J. B. Breckinridge (2004), Image Formation in High Contrast Optical Systems: The role of Polarization, SPIE Proceedings 5487 pages 1337-1345.

${ }^{8}$ R. Chipman, Polarization Aberrations, PhD Dissertation, 1987 University of Arizona, Optical Sciences Center.

${ }^{9}$ Smith, S. J. and Purcell, E. M., 1953, Phys. Rev., 92, 1069

${ }^{10}$ Haeberle, O., Rullhusen, P., Salome, J-M. and Maerne, N., 1997, Phys. Rev. E, 55, 4675

${ }^{11}$ Dirks, A. G. and Leamy, H. J., 1977, Thin Solid Films, 47, 219

${ }^{12}$ Gee, J. R. and Wilson, P. W., 1986, J. Vac. Sci. Tech., 4, 1875

${ }^{13}$ Hodgkinson, I., 1991, Appl. Opt., 30, 1303

${ }^{14}$ Windt, D. L. and Waskiewicz, W. K., 1994, J. Vac. Sci. Tech. B, 12, 3826 